\title{
Molecular Imaging of Ovarian Cancer
}

\author{
Sai Kiran Sharma ${ }^{1,2}$, Brandon Nemieboka ${ }^{3,4}$, Evis Sala $^{1,5}$, Jason S. Lewis ${ }^{1,2,4,5}$, and Brian M. Zeglis ${ }^{1,5-7}$ \\ ${ }^{1}$ Department of Radiology, Memorial Sloan Kettering Cancer Center, New York, New York; ${ }^{2}$ Program in Molecular Pharmacology, \\ Memorial Sloan Kettering Cancer Center, New York, New York; ${ }^{3}$ Tri-Institutional MD-PhD Program, New York, New York; ${ }^{4}$ Gerstner \\ Sloan Kettering Graduate School of Biomedical Sciences, Memorial Sloan Kettering Cancer Center, New York, New York; \\ ${ }^{5}$ Departments of Radiology and Pharmacology, Weill Cornell Medical College, New York, New York; ${ }^{6}$ Department of Chemistry, \\ Hunter College of City University of New York, New York, New York; and ${ }^{7}$ Graduate Center of City University of New York, New York, \\ New York
}

\begin{abstract}
Ovarian cancer is the most lethal gynecologic malignancy and the fifth leading cause of cancer-related death in women. Over the past decade, medical imaging has played an increasingly valuable role in the diagnosis, staging, and treatment planning of the disease. In this "Focus on Molecular Imaging" review, we seek to provide a brief yet informative survey of the current state of the molecular imaging of ovarian cancer. The article is divided into sections according to modality, covering recent advances in the MR, PET, SPECT, ultrasound, and optical imaging of ovarian cancer. Although primary emphasis is given to clinical studies, preclinical investigations that are particularly innovative and promising are discussed as well. Ultimately, we are hopeful that the combination of technologic innovations, novel imaging probes, and further integration of imaging into clinical protocols will lead to significant improvements in the survival rate for ovarian cancer.
\end{abstract}

Key Words: ovarian cancer; molecular imaging; PET; SPECT; ultrasound; MRI

J Nucl Med 2016; 57:827-833

DOI: 10.2967/jnumed.115.172023

Ovarian cancer continues to be the most lethal gynecologic malignancy and the fifth leading cause of cancerrelated death in women. As of 2015, the 5-y survival rate for patients with advanced-stage ovarian cancer was a dismal $27 \%$ in the United States (1). This high mortality rate can be attributed largely to the inability to clinically or radiographically detect ovarian cancer at early stages. The statistics reinforcing the importance of early detection are particularly striking. If detected at an early stage (I), there is a $90 \%$ cure rate for ovarian cancer. However, this rate drops precipitously to $20 \%-25 \%$ for patients diagnosed at later stages (III-IV) (2). The late detection of ovarian cancer originates

Received Dec. 30, 2015; revision accepted Apr. 14, 2016.

For correspondence or reprints contact: Brian M. Zeglis, Hunter College of City University of New York, 413 E. 69th St., 4th Floor, Room BB452, New York, NY 10021.

E-mail: bz102@hunter.cuny.edu

Published online Apr. 28, 2016.

COPYRIGHT (C) 2016 by the Society of Nuclear Medicine and Molecular Imaging, Inc. from the convergence of a variety of factors, including the ineffective screening tools, tests, and diagnostic methods currently used in the clinic; the vague presentation of the symptoms of the disease; the long occult period followed by the rapid doubling time of ovarian cancer; and the frequent recurrence of therapy-resistant disease after initial treatment. Clearly, advances across a variety of fields are needed to improve the diagnosis and clinical management of this disease. In the pages that follow, our goal is to focus on a growing part of this puzzle by providing a bird's-eye view of the roles that molecular imaging can play in the clinical care of ovarian cancer.

Different imaging modalities provide different types of information. Anatomic imaging methods such as ultrasound, MRI, and CT create exquisitely detailed maps of organ morphology, whereas molecular imaging modalities such as PET, SPECT, and optical imaging yield functional information on the biochemistry of tissues. Not surprisingly, both types of imaging play important roles in the clinical management of ovarian cancer. To wit, the use of ultrasound as the first imaging modality to characterize adnexal lesions and the use of CT to evaluate disease extent in patients with suspected ovarian cancer are two excellent examples of the integration of anatomic imaging into current clinical protocols. Delving slightly deeper, CT is the modality of choice for evaluating patients before initial surgery or neoadjuvant therapy and has found an important place in the staging of the disease as well $(3,4)$. Furthermore, CT has been used after primary cytoreductive surgery for the detection of residual disease, thereby serving as a critical prognostic indicator for the postoperative progression-free and overall survival of patients (5). CT may be limited in its accuracy for distinguishing recurrent disease from postoperative or postradiotherapy fibrosis. However, the sensitivity of CT for the detection of recurrent ovarian cancer improves significantly when it is combined with a functional imaging modality such as PET (6). Given that CT is a purely anatomic imaging technique, further discussion of this modality lies outside the scope of this review.

Over the past two decades, a variety of molecular imaging modalities has emerged to complement traditional anatomic imaging. Indeed, molecular imaging techniques can serve a 
variety of roles in the clinical care of ovarian cancer patients, including use to localize primary tumors; determine the extent of metastatic spread; stratify patients for surgery and therapy; evaluate the metabolic status of tumors; characterize biomarker expression by malignant tissue; and design, implement, and monitor targeted therapies. In this short review, our goal is to provide a broad overview of the roles that molecular imaging plays in the management of ovarian cancer. The review comprises 4 sections, each focused on a different imaging modality: MRI, nuclear imaging, optical imaging, and ultrasound imaging. Although primary emphasis is given to clinical studies, preclinical investigations that are particularly innovative and promising will be discussed as well.

One final clerical note before we begin: recent advances in cancer biology have revealed that the term ovarian cancer is a misnomer, a catch-all that places multiple malignancies (including those that arise from nonovarian tissues) into a single basket. As a result, it is possible that each histotype of epithelial ovarian cancer-serous, endometrioid, clear cell, and mucinous - may bear unique signature biomarkers that can be targeted for molecular imaging. It is likewise feasible that molecular imaging targeting surrogate markers of KRAS, BRAF, PI3K mutations, and TP53 mutations might be used to distinguish between indolent ovarian cancer and aggressive disease. Yet as much as we appreciate the molecular and pathophysiologic diversity of ovarian cancer, most of this knowledge is relatively new and has yet to be applied to molecular imaging. Consequently, in the pages that follow, we will yield to simplicity and expedience and use the admittedly anachronistic yet convenient term ovarian cancer.

\section{MRI}

MRI has demonstrated significant utility for ovarian cancer imaging, particularly in distinguishing between benign and malignant ovarian lesions that are indeterminate on ultrasound. For example, a retrospective study performed by Thomassin-Naggara et al. including 394 patients with indeterminate adnexal masses showed that pelvic MRI using the ADNEX MR scoring system had a sensitivity of $93.5 \%$ and specificity of $96.6 \%$ for detecting malignant tissue (7). Beyond providing anatomic information, MRI can also be used for functional imaging via diffusion-weighted MRI (DWI), dynamic contrast enhanced (DCE) MRI, and MR spectroscopy (MRS). DWI measures the Brownian motion of water molecules, which is affected by the hypercellularity and extracellular architecture of tumor tissue. Apparent diffusion coefficients derived from DWI provide a quantitative parameter of the diffusivity of the imaged tissue. In a 32-patient study, Michielsen et al. compared DWI and ${ }^{18}$ F-FDG PET/CT in the context of tumor characterization and staging $(8)$. The authors concluded that whole-body DWI had a $94 \%$ accuracy rate for primary tumor characterization and $91 \%$ accuracy for peritoneal staging. DCE MRI, on the other hand, acquires images at different time points after the injection of an MR contrast agent. The transport kinetics of the contrast agent into the tumor are calculated and depend on both the vascularity and the permeability of the malignant tissue. In a clinical study performed by Li et al., DCE MRI was able to detect a significant difference between the contrast enhancement profiles of benign and malignant ovarian tumors (9). In addition, Thomassin-Naggara et al. performed a retrospective study concluding that the addition of DWI and DCE sequences to conventional MRI can improve the diagnostic accuracy for complex adnexal masses (10).

MRS measures the chemical composition of the regions of interest to yield semiquantitative data on biochemical compounds such as choline, creatine, and lactate. Esseridou et al. evaluated MRS in 16 patients with ovarian masses who underwent MRI/MRS followed by histopathologic examination of the masses (11). Of the 19 malignant tumors identified by histopathology, 17 demonstrated a choline peak on MRS, indicative of metabolic deregulation and malignancy. Multiparametric MRI can also provide insights into tumor heterogeneity, and quantitative parameters derived from DCE MRI, DWI, and MRS may serve as treatment response biomarkers in patients with advanced ovarian cancer. In a prospective study of 21 patients undergoing neoadjuvant chemotherapy for advanced ovarian cancer, Sala et al. found significant differences in baseline apparent diffusion coefficients between primary ovarian cancer, omental caking, and peritoneal deposits, indicating that diffusivity profiles may be tumor site-dependent and suggestive of the biologic heterogeneity of the disease (Fig. 1) (12). In this work, apparent diffusion coefficients and the fractional volume of the extravascular extracellular space correlated with the cytotoxic effects of platinum-based therapy and are thus potentially useful biomarkers of response. In contrast, changes in choline concentration predicted but did not reflect response. Furthermore, ovarian tumors and metastatic peritoneal implants displayed spatial heterogeneity, as perfusion, diffusion, and metabolism can vary markedly between spatially distant tumor subregions. This variation likely reflects distinct phenotypic habitats within the tumor, distinct clonality, or a combination thereof.

\section{NUCLEAR IMAGING}

Not surprisingly, the most ubiquitous radiotracer in oncologic PET imaging - ${ }^{18} \mathrm{~F}-\mathrm{FDG}$ - has been used in a variety of ways in the management of ovarian cancer. Several reports point to the superior performance of ${ }^{18} \mathrm{~F}$-FDG PET/CT in the detection of recurrent ovarian cancer. ${ }^{18} \mathrm{~F}-\mathrm{FDG}$ PET/CT is reported to have found positive lesions in many instances in which CT alone was negative (13). Furthermore, ${ }^{18} \mathrm{~F}-\mathrm{FDG}$ PET was able to document the evidence of disease recurrence 6 mo before findings could be seen on CT (14). Preoperative whole-body imaging via ${ }^{18} \mathrm{~F}-\mathrm{FDG} \mathrm{PET} / \mathrm{CT}$ has often contributed to the accurate upstaging of ovarian cancer patients as well, especially with regard to lymph node involvement (Fig. 2) $(15,16)$. In addition to the qualitative visualization of lesions on an ${ }^{18} \mathrm{~F}-\mathrm{FDG}$ PET scan, semiquantitative 


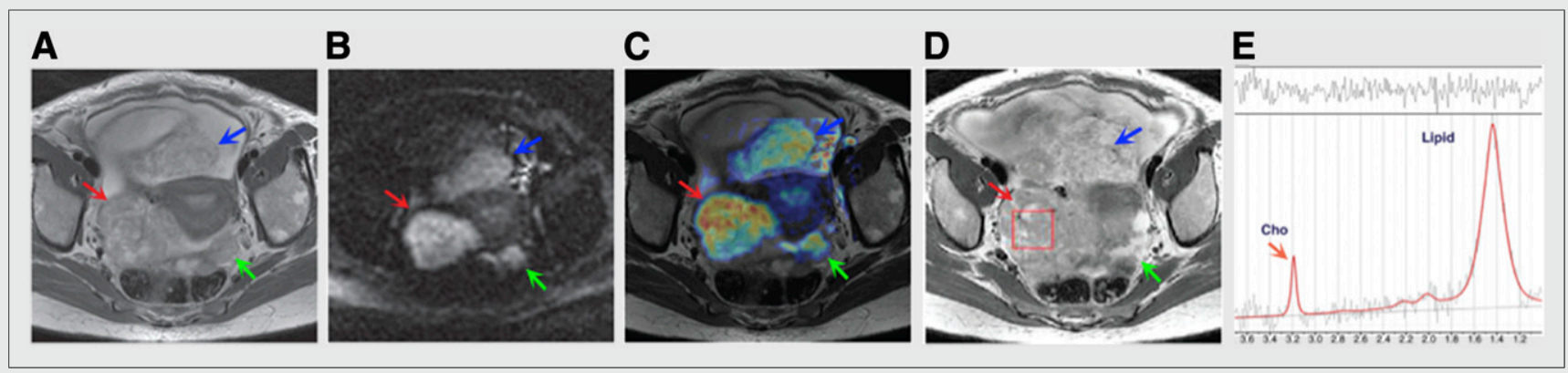

FIGURE 1. Multiparametric pretreatment MR images (3.0 T) of primary ovarian tumor (red arrows), omental cake (blue arrows), and peritoneal implant (green arrows) in 58-y-old woman with advanced ovarian cancer: T2-weighted image (A); diffusion-weighted image $\left(b=500 \mathrm{~s} / \mathrm{mm}^{2}\right)(B)$; fused T2-weighted and diffusion-weighted image (C); T2-weighted image showing region of interest for MRS (red) from primary tumor (D); spectral fit (red) overlaid on raw MRS data (gray), illustrating strong choline (Cho) signal (E). (Adapted and reprinted with permission of (12).)

parameters such as SUV have been shown to have prognostic and predictive utility during clinical follow-up (17). Along these lines, Vallius et al. used ${ }^{18} \mathrm{~F}$-FDG PET/CT to predict histopathologic responders and nonresponders to neoadjuvant chemotherapy before interval debulking surgery (18). In another example, a recent clinical study in 12 platinum-resistant patients demonstrated an exposure-response relationship between a pan-Akt inhibitor and the tumoral uptake of ${ }^{18}$ F-FDG (19).

Despite its utility in ovarian cancer, ${ }^{18} \mathrm{~F}-\mathrm{FDG}$ PET has some important limitations, most notably the prevalence of false negatives associated with the cystic nature of ovarian cancer

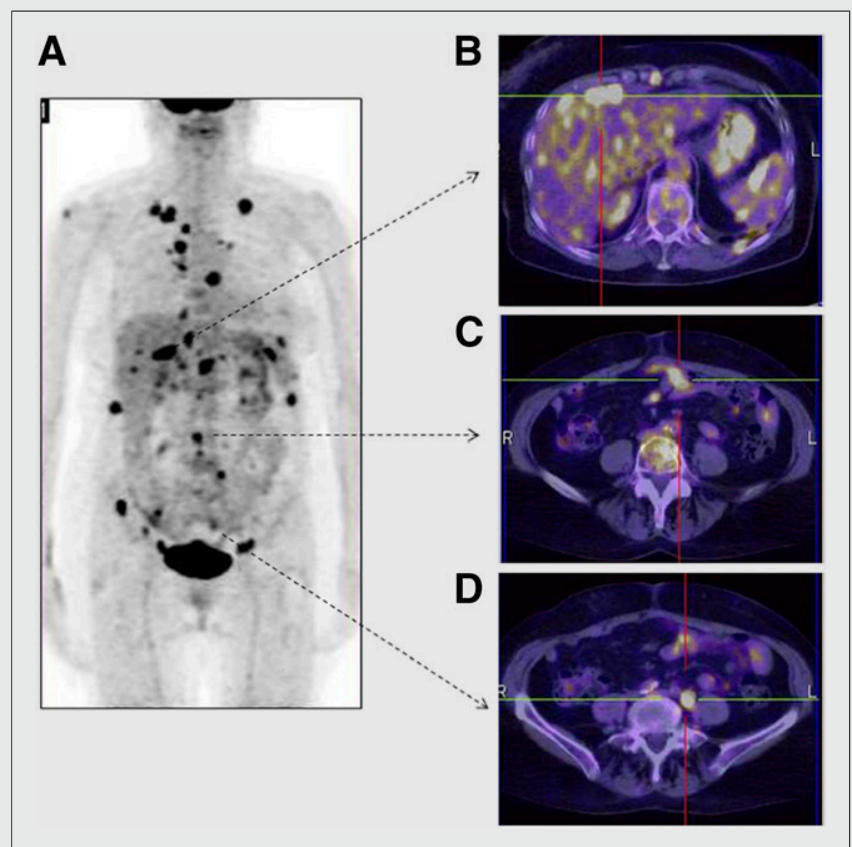

FIGURE 2. ${ }^{18} \mathrm{~F}-\mathrm{FDG}$ PET/CT images of recurrent ovarian cancer 10 mo after treatment with radical surgery and adjuvant chemotherapy: maximum-intensity projection $(A)$ and transaxial images delineating uptake of radiotracer in liver $(B)$, peritoneum (C), and locoregional lymph nodes (D). (Adapted and reprinted with permission of (16).) and false positives stemming from the uptake of ${ }^{18} \mathrm{~F}-\mathrm{FDG}$ in inflammatory cells and benign growths. In response to these limitations, an impressive array of second-generation radiotracers has been developed and translated to the clinic. Generally speaking, these radiotracers target cell-surface receptors that are overexpressed in ovarian cancer. The putative clinical advantage of these molecular imaging strategies lies in the ability to provide noninvasive, real-time, and quantitative whole-body information on the in vivo receptor status of ovarian tumors. This information can then be leveraged to facilitate the stratification, planning, or monitoring of receptortargeted therapies. For example, two SPECT radiotracers that target the folate receptor- ${ }^{111}$ In-DTPA-folate and ${ }^{99 \mathrm{~m}} \mathrm{Tc}-$ etarfolatide - have shown promise for the delineation of newly diagnosed ovarian cancer and the stratification of patients based on their levels of folate receptor expression in ovarian tumor lesions, respectively $(20,21)$. Shifting gears to PET, a recent clinical report probing the possibility of imaging estrogen receptor- $\alpha$ levels with ${ }^{18}$ F-labeled estradiol found that this radiotracer could delineate estrogen receptor- $\alpha$-positive from -negative tumors with high specificity (22). Similarly, mesothelin is overexpressed in ovarian cancer and is known to interact synergistically with MUC16 to promote the peritoneal seeding and spread of ovarian cancer. A recent firstin-human PET imaging study using an ${ }^{89} \mathrm{Zr}$-labeled antimesothelin antibody in 4 ovarian cancer and 7 pancreatic cancer patients found that the mean $\mathrm{SUV}_{\max }$ was higher in ovarian cancer lesions than in pancreatic cancer lesions (23). This approach could help in the identification of metastatic ovarian cancer in patients who may benefit from treatment with antimesothelin therapy.

Moving from the clinic to the laboratory, several promising imaging agents have been the subject of preclinical studies in recent years. Sharma et al., for example, have reported the development of an ${ }^{89} \mathrm{Zr}$-labeled radioimmunoconjugate based on the CA125-targeting murine antibody B43.13 (24). Notably, this agent is capable of delineating the metastatic spread of OVCAR3 tumors along the ipsilateral chain of lymph nodes. Ocak et al. recently used a ${ }^{68} \mathrm{Ga}$-DOTA-albumin-folate conjugate and FR680 for the multimodal PET/fluorescence 
molecular tomographic imaging of the intraperitoneal spread of folate receptor-positive MKP-L cells (25). Similarly, Liu et al. reported on the multimodal PET/optical imaging of metastatic ovarian tumors within the peritoneum of mice using a ${ }^{64} \mathrm{Cu}$-labeled pyropheophorbide-folate conjugate (26). Such multimodal molecular imaging agents could be clinically useful both for the preoperative delineation of the extent of disease and as intraoperative tools for the efficient resection of residual disease during cytoreductive surgery. On a different note, an increasing understanding of the roles played by components of the immune system-for example, macrophages and cytokines-in the promotion of tumor growth has led to the emergence of yet another class of targets for molecular imaging. Along these lines, Li et al. have recently created a SPECT imaging agent to target the interleukin-6 receptor (IL-6R) (9). In their work, a radiolabeled IL-6Rtargeting peptide- ${ }^{99 m}$ Tc-HYNIC-Aca-LSLITRL_-displayed increased uptake in $\mathrm{C} 13 \mathrm{~K}$ tumors, which express high levels of IL-6R, and much reduced retention in SKOV3.ip tumors, which express low levels of IL-6R. In theory, molecular imaging of the IL-6R status of tumors could aid in the identification of patients who may benefit from IL-6/IL-6R therapies.

A particularly interesting subset of preclinical investigations has focused on the use of nuclear imaging for the monitoring of therapeutic progress. For example, ${ }^{99 m}$ Tc-peptide- $Z_{\text {HER2:342, }}$ an Affibody that targets human epidermal growth factor receptor 2 (HER2), recently was successfully used to monitor the efficacy of trastuzumab therapy in a SKOV3 xenograft model via SPECT (27). Likewise, Niu et al. have shown that the uptake of ${ }^{64} \mathrm{Cu}$-DOTA-trastuzumab can be used to monitor response to therapy with 17-dimethylaminoethylamino17-demethoxygeldanamycin (28). Finally, Nagengast et al. have demonstrated the utility of ${ }^{89} \mathrm{Zr}$-labeled bevacizumab as an imaging marker for early response to antiangiogenic therapy with an Hsp90 inhibitor, NVP-AUY922 (29).

\section{OPTICAL IMAGING}

Optical imaging is emerging as a powerful tool in biomedical research and clinical practice. In oncology, the use of fluorescent probes as tools for image-guided surgery has proven particularly promising. In this setting, the imaging agents assist surgeons in distinguishing malignant from benign tissue with the help of a navigation system that activates the molecular probe and captures and processes the emitted light to generate an image in real time (30). The first-in-human studies using this approach were published in 2011 by van Dam et al., who exploited the overexpression of folate receptor- $\alpha$ in ovarian cancer by developing a conjugated fluorescein isothiocyanate-folate probe (Fig. 3) (31). Ten patients with suspected ovarian cancer received an injection of the probe and underwent image-guided surgery followed by postoperative histopathologic analyses. Critically, all tissue samples that fluoresced in vivo and ex vivo were confirmed to be malignant, whereas none of the resected benign tissues exhibited fluorescence. Similarly, a clinical trial is currently under way with data recently published by Tummers et al. (32). Briefly, $\mathrm{EC} 17$, an analog of the conjugated fluorescein isothiocyanatefolate probe, was injected into patients undergoing cytoreductive surgery. From the 12 ovarian cancer patients, 44 confirmed malignant lesions were resected, including 7 that were not identified by initial surgical inspection. Recently, the attention of the field has shifted from fluorophores that emit visible light to probes that emit near-infrared fluorescence. In the clinic, Tummers et al. conducted a trial in which 10 patients with suspected ovarian cancer were administered indocyanine green (emission wavelength, $\sim 830 \mathrm{~nm}$ ) before imageguided cytoreductive surgery. Although all metastatic deposits in these patients exhibited near-infrared fluorescence, 13 nonmalignant lesions also exhibited fluorescence, leading to a high false-positive rate of $62 \%$ (33). Preclinical advances in near-infrared fluorescence imaging have been made as well.

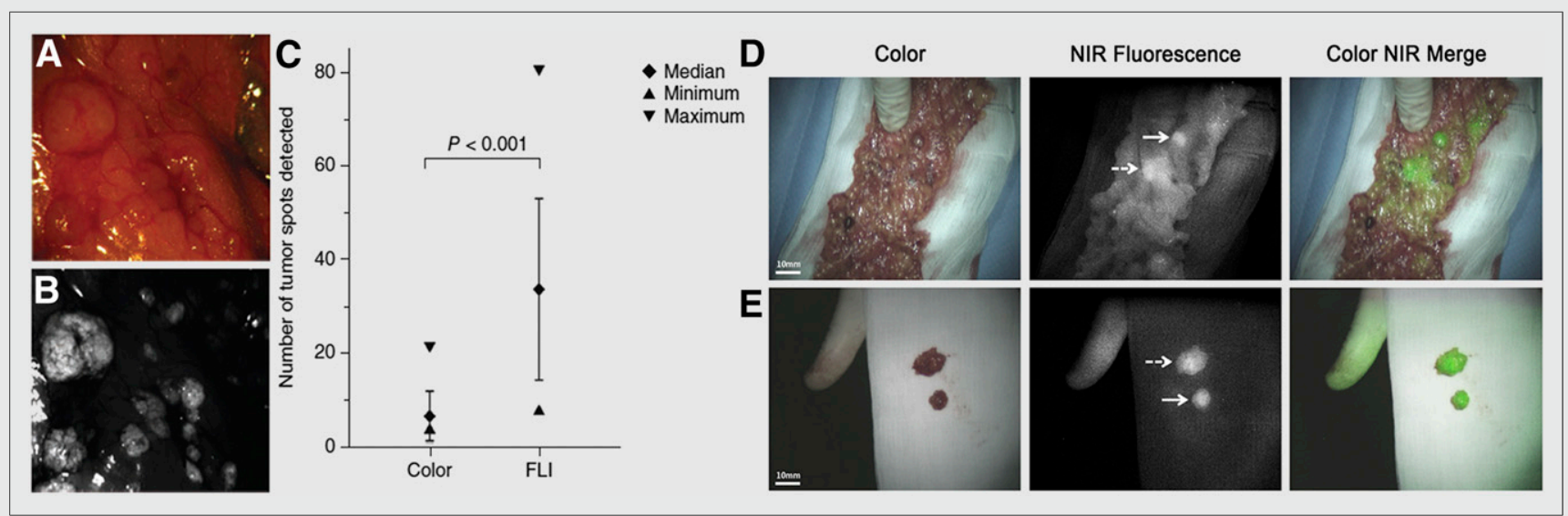

FIGURE 3. Identification of metastatic ovarian cancer deposits via intraoperative and ex vivo fluorescence imaging. (A and B) Comparative images of tumors within abdominal cavity visualized under white light (A) vs. fluorescence emissions from folate receptor-targeted probe (B). (C) Statistical analysis revealing benefit of intraoperative fluorescence imaging. (D) Identification of two metastatic lesions in greater omentum using indocyanine green dye for intraoperative near-infrared (NIR) fluorescence imaging. (E) Ex vivo visualization of resected metastatic lesions. (Adapted and reprinted with permission of $(31,33)$.) 


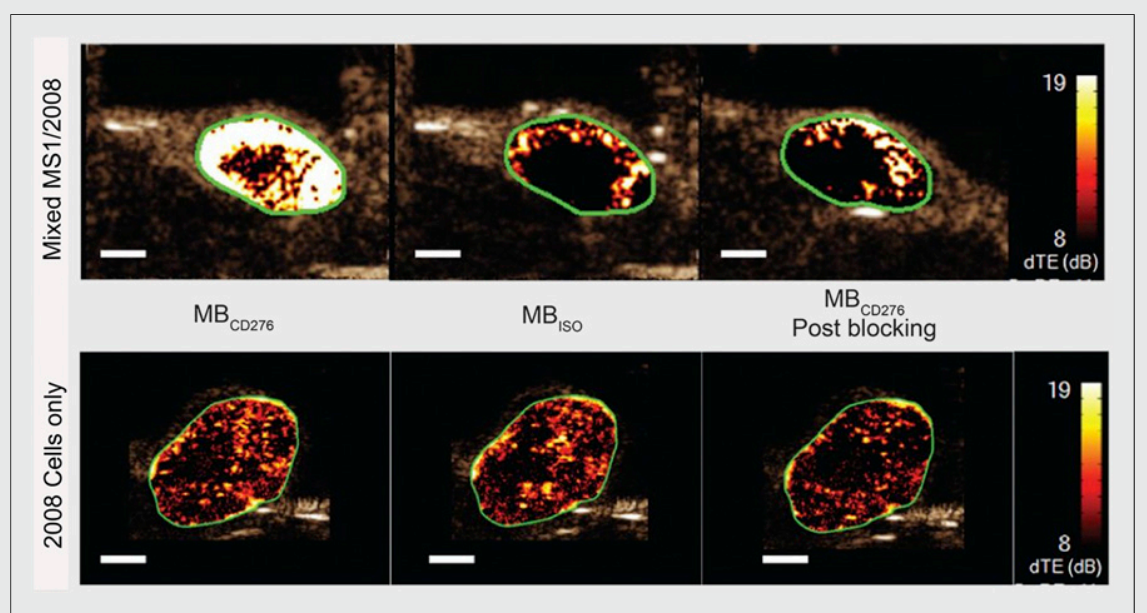

FIGURE 4. Preclinical contrast-enhanced ultrasound imaging of angiogenesis. Microbubbles functionalized with CD276-targeting antibody ( $\left.\mathrm{MB}_{\mathrm{CD} 276}\right)$ effectively targeted subcutaneous xenografts comprising 2008 human endometrioid ovarian cancer cells mixed with CD276-expressing MS1 mouse endothelial cells. Specificity of targeting was demonstrated via comparison with isotype antibody-functionalized microbubbles $\left(\mathrm{MB}_{\text {ISO }}\right)$ as well as blocking experiment in which excess of CD276 antibody was administered. (Adapted and reprinted with permission of (42).)

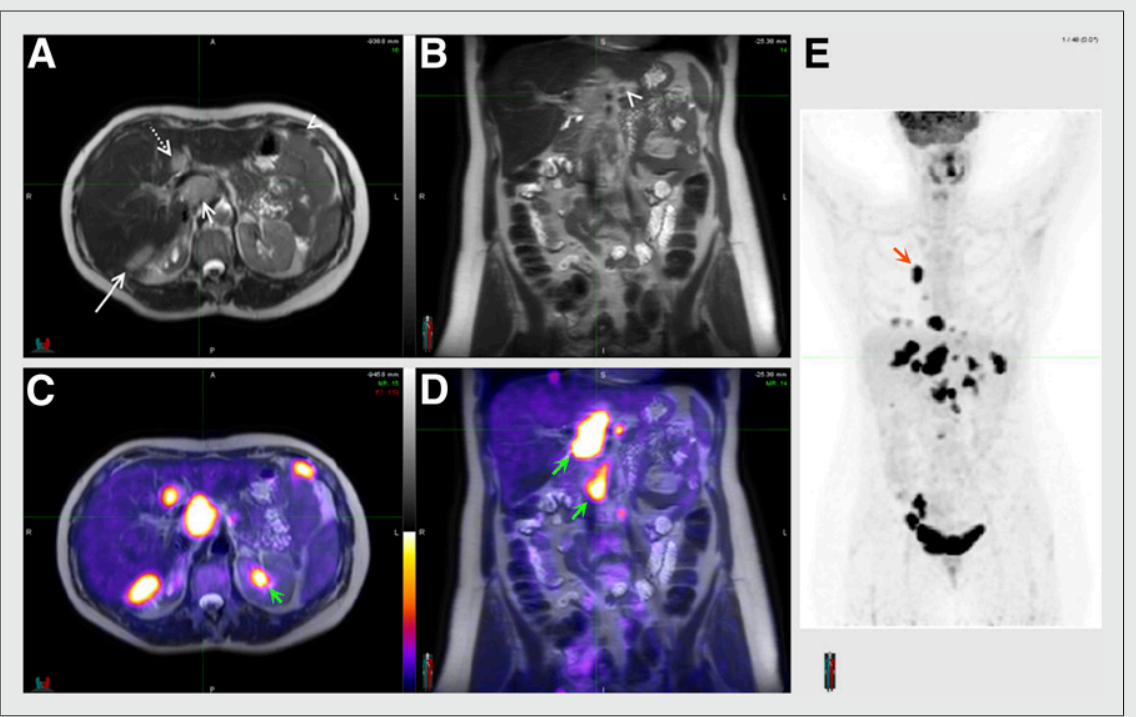

FIGURE 5. PET/MRI, an emerging hybrid imaging technique: axial $(A)$ and coronal (B) T2-weighted MRI scans of ovarian cancer patient, in which tumor lesions were seen adjacent to liver (long solid arrow), in segment IV of liver (dotted arrow), in porta hepatis (short solid arrow), and in peritoneum (arrowheads); axial (C) and coronal (D) PET/MRI scans wherein ${ }^{18} \mathrm{~F}$-FDG PET not only demonstrated excellent correlation with lesions identified previously by MRI alone but also highlighted new lesions (arrows); (E) whole-body maximum-intensity projection from ${ }^{18} \mathrm{~F}$-FDG PET showing multiple lesions in chest (arrow) and abdomen. (Adapted and reprinted with permission of (43).)

To wit, Terwisscha van Scheltinga et al. targeted HER2 and vascular endothelial growth factor using IRDye $800 \mathrm{CW}-$ labeled monoclonal antibodies (34), whereas Lee et al. targeted folate receptor- $\alpha$ with an activatable near-infrared fluorescence probe that emits fluorescence only after cleavage of a linker by the lysosomal enzyme cathepsin B (35).

\section{ULTRASOUND}

Both photoacoustic imaging and traditional ultrasound have also shown promise for ovarian cancer imaging. As its name suggests, photoacoustic imaging is predicated on the photoacoustic effect: the generation of ultrasonic waves after the absorption of photons by biomolecules such as hemoglobin or melanin. Because scattering in biologic tissue is lower for ultrasonic waves than for photons, photoacoustic imaging has the potential to visualize tumors deep within the body (36). Aguirre et al. have developed a coregistered ultrasound/photoacoustic imaging system and used it to image 33 ovary samples collected from patients undergoing oophorectomy, ultimately determining a sensitivity and specificity of $83 \%$ for diagnosing ovarian cancer in postmenopausal ovaries (37). More recently, several preclinical papers have been published improving on the hybrid technology, including the creation of a system for the real-time coregistration of images (38) and a miniaturized illumination probe for transvaginal photoacoustic imaging (39). Exogenous contrast agents can also be used for photoacoustic imaging, as Jokerst et al. have illustrated in their work using nontargeted gold nanorods as contrast agents for photoacoustic and Raman imaging (40).

Traditional ultrasound continues to be further improved as well. Specifically, several promising preclinical investigations have shed light on the potential of microbubble-based contrast agents for the imaging of tumor angiogenesis. For example, Willmann et al. observed increased ultrasound signal in ovarian tumor tissue after the administration of perfluorocarbon-filled microbubbles conjugated to cystine knot (knottin) peptides targeting integrin $\alpha_{\mathrm{V}} \beta_{3}(41)$. More recently, Lutz et al. observed a significantly higher ultrasound signal using microbubbles engineered to target the vascular marker CD276 (Fig. 4) (42). Ultimately, the realtime acquisition and processing of both traditional ultrasound and photoacoustic imaging — combined with the fact that ultrasound systems are inexpensive and widespread in the clinic-make these modalities primed for increased use as diagnostic tools for ovarian cancer. 


\section{EMERGING INNOVATIONS}

There are a variety of different avenues that are primed for innovation. In our opinion, two stand out. First, the use of hybrid imaging systems - such as PET/MR - that provide simultaneous anatomic and functional data has shown potential in the ovarian cancer setting and certainly merits further investigation (Fig. 5). Second, the current push to unravel the molecular fingerprints of the subtypes of ovarian cancer has yielded a list of new biomarkers that is growing by the day. Molecular imaging agents targeting these biomarkers have the potential to serve two exciting roles: as diagnostic tools for the identification of a patient's tumor type and as theranostic tools for the selection of personalized targeted therapies.

\section{CONCLUSION}

With the advent of personalized medicine, the field of ovarian cancer research has reached a fascinating crossroads. Much of the silence of the "silent killer" has been dispelled, and recent years have witnessed a surge in the understanding of the etiology and molecular characteristics of the disease. In light of these advances, we believe that imaging will play a central role in both the preclinical study and the clinical management of ovarian cancer in the years to come. As we have discussed in these pages, an array of extremely promising imaging strategies has already had an impact on clinical care, and the future is even brighter. In the end, we are optimistic and hopeful that the combination of technologic innovations, novel imaging probes, and further integration of imaging into clinical protocols will lead to significant and lasting improvements in the prognosis and care of ovarian cancer patients.

\section{DISCLOSURE}

The authors' work is supported by the Tow Foundation, the NIH (4R00CA178205-02 to Brian M. Zeglis and 3R01CA176671-02S1 to Jason S. Lewis and Brandon Nemieboka), by William H. Goodwin, Jr., and Alice T. Goodwin and their Commonwealth Foundation for Cancer Research, by the Center for Experimental Therapeutics at Memorial Sloan Kettering Cancer Center, and by a support grant from the Memorial Sloan Kettering Cancer Center (P30 CA008748). No other potential conflict of interest relevant to this article was reported.

\section{REFERENCES}

1. Siegel RL, Miller KD, Jemal A. Cancer statistics, 2015. CA Cancer J Clin. 2015;65:5-29.

2. Gupta D, Lis CG. Role of CA125 in predicting ovarian cancer survival: a review of the epidemiological literature. J Ovarian Res. 2009;2:13.

3. Glaser G, Torres M, Kim BH, et al. The use of CT findings to predict extent of tumor at primary surgery for ovarian cancer. Gynecol Oncol. 2013;130:280-283.

4. Iyer VR, Lee SI. MRI, CT, and PET/CT for ovarian cancer detection and adnexal lesion characterization. AJR. 2010;194:311-321.

5. Burger IA, Goldman DA, Vargas HA, et al. Incorporation of postoperative CT data into clinical models to predict 5-year overall and recurrence free survival after primary cytoreductive surgery for advanced ovarian cancer. Gynecol Oncol. 2015;138:554-559.

6. Gu P, Pan LL, Wu SQ, Sun L, Huang G. CA 125, PET alone, PET-CT, CT and MRI in diagnosing recurrent ovarian carcinoma: a systematic review and metaanalysis. Eur J Radiol. 2009;71:164-174.

7. Thomassin-Naggara I, Aubert E, Rockall A, et al. Adnexal masses: development and preliminary validation of an MR imaging scoring system. Radiology. 2013; 267:432-443

8. Michielsen K, Vergote I, Op de Beeck K, et al. Whole-body MRI with diffusionweighted sequence for staging of patients with suspected ovarian cancer: a clinical feasibility study in comparison to CT and FDG-PET/CT. Eur Radiol. 2014;24:889-901.

9. Li X, Hu JL, Zhu LM, et al. The clinical value of dynamic contrast-enhanced MRI in differential diagnosis of malignant and benign ovarian lesions. Tumour Biol. 2015;36:5515-5522.

10. Thomassin-Naggara I, Toussaint I, Perrot N, et al. Characterization of complex adnexal masses: value of adding perfusion- and diffusion-weighted MR imaging to conventional MR imaging. Radiology. 2011;258:793-803.

11. Esseridou A, Di Leo G, Sconfienza LM, et al. In vivo detection of choline in ovarian tumors using 3D magnetic resonance spectroscopy. Invest Radiol. 2011; 46:377-382

12. Sala E, Kataoka MY, Priest AN, et al. Advanced ovarian cancer: multiparametric MR imaging demonstrates response- and metastasis-specific effects. Radiology. 2012;263:149-159.

13. Thrall MM, DeLoia JA, Gallion H, Avril N. Clinical use of combined positron emission tomography and computed tomography (FDG-PET/CT) in recurrent ovarian cancer. Gynecol Oncol. 2007;105:17-22.

14. Zimny M, Siggelkow W, Schroder W, et al. 2-[fluorine-18]-fluoro-2-deoxyd-glucose positron emission tomography in the diagnosis of recurrent ovarian cancer. Gynecol Oncol. 2001;83:310-315.

15. Kyriazi S, Kaye SB, deSouza NM. Imaging ovarian cancer and peritoneal metastases: current and emerging techniques. Nat Rev Clin Oncol. 2010;7:381-393.

16. Caobelli F, Alongi P, Evangelista L, et al. Predictive value of ${ }^{18} \mathrm{~F}-\mathrm{FDG}$ PET/CT in restaging patients affected by ovarian carcinoma: a multicentre study. Eur $J$ Nucl Med Mol Imaging. 2016;43:404-413.

17. Sala E, Kataoka M, Pandit-Taskar N, et al. Recurrent ovarian cancer: use of contrast-enhanced CT and PET/CT to accurately localize tumor recurrence and to predict patients' survival. Radiology. 2010;257:125-134.

18. Vallius T, Peter A, Auranen A, et al. F-FDG-PET/CT can identify histopathological non-responders to platinum-based neoadjuvant chemotherapy in advanced epithelial ovarian cancer. Gynecol Oncol. 2016;140:29-35.

19. Gungor H, Saleem A, Babar S, et al. Dose-finding quantitative FDG PET imaging study with the oral pan-AKT inhibitor GSK2141795 in patients with gynecological malignancies. J Nucl Med. 2015;56:1828-1835.

20. Siegel BA, Dehdashti F, Mutch DG, et al. Evaluation of ${ }^{111}$ In-DTPA-folate as a receptor-targeted diagnostic agent for ovarian cancer: initial clinical results. J Nucl Med. 2003;44:700-707.

21. Morris RT, Joyrich RN, Naumann RW, et al. Phase II study of treatment of advanced ovarian cancer with folate-receptor-targeted therapeutic (vintafolide) and companion SPECT-based imaging agent ( ${ }^{99 \mathrm{~m}}$ Tc-etarfolatide). Ann Oncol. 2014;25:852-858.

22. van Kruchten M, de Vries EF, Arts HJ, et al. Assessment of estrogen receptor expression in epithelial ovarian cancer patients using $16 \alpha^{-18} \mathrm{~F}$-fluoro-17 $\beta$ estradiol PET/CT. J Nucl Med. 2015;56:50-55.

23. Lamberts LE, Menke-van der Houven van Oordt CW, Ter Weele EJ, et al. ImmunoPET with anti-mesothelin antibody in patients with pancreatic and ovarian cancer before anti-mesothelin antibody-drug conjugate treatment. Clin Cancer Res. 2016;22:1642-1652.

24. Sharma SK, Sevak KK, Monette S, et al. Preclinical ${ }^{89} \mathrm{Zr}$-immunoPET of high grade serous ovarian cancer and lymph node metastasis. J Nucl Med. February 2, 2016 [Epub ahead of print].

25. Ocak M, Gillman AG, Bresee J, et al. Folate receptor-targeted multimodality imaging of ovarian cancer in a novel syngeneic mouse model. Mol Pharm. 2015;12:542-553.

26. Liu TW, Stewart JM, Macdonald TD, et al. Biologically-targeted detection of primary and micro-metastatic ovarian cancer. Theranostics. 2013;3:420-427.

27. Zhang J, Zhao X, Wang S, et al. Monitoring therapeutic response of human ovarian cancer to trastuzumab by SPECT imaging with ${ }^{99 m}$ Tc-peptide-Z(HER2:342). Nucl Med Biol. 2015;42:541-546.

28. Niu G, Li Z, Cao Q, Chen X. Monitoring therapeutic response of human ovarian cancer to 17-DMAG by noninvasive PET imaging with ${ }^{64} \mathrm{Cu}$-DOTA-trastuzumab. Eur J Nucl Med Mol Imaging. 2009;36:1510-1519.

29. Nagengast WB, de Korte MA, Oude Munnink TH, et al. ${ }^{89} \mathrm{Zr}$-bevacizumab PET of early antiangiogenic tumor response to treatment with HSP90 inhibitor NVPAUY922. J Nucl Med. 2010;51:761-767. 
30. Chi C, Du Y, Ye J, et al. Intraoperative imaging-guided cancer surgery: from current fluorescence molecular imaging methods to future multi-modality imaging technology. Theranostics. 2014;4:1072-1084.

31. van Dam GM, Themelis G, Crane LM, et al. Intraoperative tumor-specific fluorescence imaging in ovarian cancer by folate receptor-alpha targeting: first in-human results. Nat Med. 2011;17:1315-1319.

32. Tummers QJ, Hoogstins CE, Gaarenstroom KN, et al. Intraoperative imaging of folate receptor alpha positive ovarian and breast cancer using the tumor specific agent EC17. Oncotarget. March 22, 2016 [Epub ahead of print].

33. Tummers QR, Hoogstins CE, Peters AA, et al. The value of intraoperative near-infrared fluorescence imaging based on enhanced permeability and retention of indocyanine green: feasibility and false-positives in ovarian cancer. PLoS One. 2015;10:e0129766.

34. Terwisscha van Scheltinga AG, van Dam GM, Nagengast WB, et al. Intraoperative near-infrared fluorescence tumor imaging with vascular endothelial growth factor and human epidermal growth factor receptor 2 targeting antibodies. $\mathrm{J} \mathrm{Nucl} \mathrm{Med}$. 2011;52:1778-1785.

35. Lee H, Kim J, Kim H, Kim Y, Choi Y. A folate receptor-specific activatable probe for near-infrared fluorescence imaging of ovarian cancer. Chem Commun (Camb). 2014;50:7507-7510.

36. Mallidi S, Luke GP, Emelianov S. Photoacoustic imaging in cancer detection, diagnosis, and treatment guidance. Trends Biotechnol. 2011;29:213-221.
37. Aguirre A, Ardeshirpour Y, Sanders MM, Brewer M, Zhu Q. Potential role of coregistered photoacoustic and ultrasound imaging in ovarian cancer detection and characterization. Transl Oncol. 2011;4:29-37.

38. Alqasemi U, Li H, Yuan G, Kumavor P, Zanganeh S, Zhu Q. Interlaced photoacoustic and ultrasound imaging system with real-time coregistration for ovarian tissue characterization. J Biomed Opt. 2014;19:76020.

39. Salehi HS, Wang T, Kumavor PD, Li H, Zhu Q. Design of miniaturized illumination for transvaginal co-registered photoacoustic and ultrasound imaging. Biomed Opt Express. 2014;5:3074-3079.

40. Jokerst JV, Cole AJ, Van de Sompel D, Gambhir SS. Gold nanorods for ovarian cancer detection with photoacoustic imaging and resection guidance via Raman imaging in living mice. ACS Nano. 2012;6:10366-10377.

41. Willmann JK, Kimura RH, Deshpande N, Lutz AM, Cochran JR, Gambhir SS. Targeted contrast-enhanced ultrasound imaging of tumor angiogenesis with contrast microbubbles conjugated to integrin-binding knottin peptides. $\mathrm{J} \mathrm{Nucl}$ Med. 2010;51:433-440.

42. Lutz AM, Bachawal SV, Drescher CW, Pysz MA, Willmann JK, Gambhir SS. Ultrasound molecular imaging in a human CD276 expression-modulated murine ovarian cancer model. Clin Cancer Res. 2014;20:1313-1322.

43. Partovi S, Kohan A, Rubbert C, et al. Clinical oncologic applications of PET/MRI: a new horizon. Am J Nucl Med Mol Imaging. 2014;4:202-212. 